\title{
Solución numérica de las ecuaciones estocásticas de la cinética puntual usando el esquema Runge-Kutta implícito de orden 1.5
}

\author{
Daniel Suescún-Díaz, Luis E. Arévalo y Diego A. Rasero \\ Departamento de Ciencias Naturales, Universidad Surcolombiana, Neiva, Huila, Colombia. \\ (correo-e: daniel.suescun@usco.edu.co, lulabmx-94@hotmail.com, diego.rasero@usco.edu.co)
}

Recibido Mar. 30, 2021; Aceptado May. 28, 2021; Versión final Jun. 22, 2021, Publicado Dic. 2021

\section{Resumen}

En este trabajo se propone el esquema de Runge-Kutta implícito de orden 1.5 para solucionar de forma numérica las ecuaciones de la cinética puntual estocástica, considerando diferentes valores de reactividad. Se obtiene la matriz de covarianza usando la teoría de difusión de una velocidad para un reactor nuclear. Para esto, se asume que en el tiempo inicial un pulso de neutrones es inyectado en un ensamble no multiplicativo. Los resultados obtenidos con este método logran una buena precisión en la desviación estándar y el valor esperado en comparación con otros métodos reportados en la literatura. Se concluye que el método propuesto es eficiente para el cálculo de la densidad de población de neutrones y la concentración de precursores de neutrones atrasados.

Palabras clave: ecuaciones estocásticas; cinética puntual; reactor nuclear; densidad; neutrones; precursor; Runge-Kutta

\section{Numerical solution for stochastic point kinetic equations using the implicit order 1.5 Runge-Kutta scheme}

\begin{abstract}
In the present study, the implicit order 1.5 Runge-Kutta scheme is proposed to numerically solve stochastic point kinetic equations by considering different reactivity values. A covariance matrix is obtained by applying the one-velocity diffusion theory for a nuclear reactor. It is assumed that at initial time a neutron pulse is injected into a non-multiplicative assembly. The results obtained by applying this method are accurate, based on the standard deviation and the expected value, when compared to other methods reported in the literature. It is concluded that the proposed method is accurate for calculating neutron density and delayed neutron precursor concentration.
\end{abstract}




\section{INTRODUCCIÓN}

Las ecuaciones de la cinética puntual son un sistema de ecuaciones diferenciales no lineales y fuertemente acopladas, las cuales modelan la evolución en el tiempo de la densidad de neutrones y la concentración de precursores de neutrones atrasados en un reactor nuclear, su solución puede dar información del comportamiento de la potencia del reactor durante el encendido, el apagado y el movimiento de las barras de control. Estas ecuaciones son determinísticas, pero se ha evidenciado que el comportamiento de la potencia del reactor es aleatorio (Stacey, 2018). Así, es necesario describir la evolución en el tiempo de la densidad de neutrones y la concentración de precursores como un proceso estocástico, representado como un conjunto de ecuaciones diferenciales estocásticas. El problema de las ecuaciones de la cinética puntual estocástica ha sido resuelto, asumiendo que la variación en la población de neutrones sigue una distribución normal.

Entre los métodos reportados están: la aproximación constante por partes (PCA) y Monte Carlo (Hayes y Allen, 2005), Euler-Maruyama explícito y Taylor 1.5 (Ray, 2012 ; Ray y Patra, 2013), el método de la cinética puntual estocástica simplificado (SSPK) (Ayyoubzadeh y Vosoughi, 2014), el modelo exponencial analítico (AEM) (Nahla y Edress, 2016a), el modelo estocástico eficiente (ESM) (Nahla y Edress, 2016b), el método de doble diagonalización y descomposición (DDDM) (Da Silva et al., 2016), el método usando lógica difusa (Nayak y Chakraverty, 2016), el método de pasos divididos (Singh y Ray, 2017), el método de Euler-Maryama implícito (Suescún-Díaz et al., 2018), el método de expansión Wiener-Hermite (EWH) (El-Beltagy y Noor, 2019) y finalmente se encuentra el método de validación (Gordon et al., 2021).

En este trabajo se propone el método de Runge-Kutta implícito de orden 1.5, para implementar el método se utiliza la matriz de covarianza modificada, la cual se obtiene de la teoría de difusión de una velocidad considerando los neutrones pulsados (Suescún-Díaz et al., 2018). El método propuesto hace uso de la solución analítica de la matriz inversa esto permite un menor costo computacional en los distintos experimentos numéricos con reactividades constantes. Este trabajo está organizado de la siguiente forma, inicialmente se presenta las consideraciones teóricas, en la siguiente sección el método implícito estocástico de Runge-Kutta, en la siguiente sección el análisis de resultados y finalmente las conclusiones.

Las ecuaciones de la cinética puntual son un sistema de ecuaciones diferenciales no lineales y fuertemente acopladas que modelan la dinámica de un reactor nuclear, estas ecuaciones son deterministas y solo estiman el valor medio de la densidad de neutrones y la concentración de precursores de neutrones atrasados, sin embargo, considerando que en la práctica se presentan fluctuaciones en la potencia de un rector nuclear y existen diferentes posibilidades para el estado de la población de neutrones, se debe considerar la dinámica de la población de neutrones como un proceso estocástico (Hayes y Allen, 2005), de esta manera el sistema de las ecuaciones estocásticas de la cinética puntual puede escribirse brevemente de la siguiente manera:

$$
\frac{\mathbf{d} \widehat{\mathbf{P}}(\mathbf{t})}{\mathbf{d t}}=\mathbf{A} \widehat{\mathbf{P}}(\mathbf{t})+\widehat{\mathbf{Q}}(\mathbf{t})+\widehat{B}^{1 / 2} \frac{\mathbf{d} \widehat{\mathbf{W}}}{\mathbf{d t}}
$$

Donde todas las variables que dependen del tiempo son variables aleatorias y están representadas por:

$$
\widehat{P}(\mathrm{t})=\left[\begin{array}{c}
n(t) \\
C_{1}(t) \\
\vdots \\
C_{m}(t)
\end{array}\right], \quad \widehat{Q}(\mathrm{t})=\left[\begin{array}{c}
q(t) \\
0 \\
\vdots \\
0
\end{array}\right], \quad \widehat{W}(\mathrm{t})=\left[\begin{array}{c}
W_{1}(t) \\
W_{2}(t) \\
\vdots \\
W_{m+1}(t)
\end{array}\right]
$$

Siendo $m$ el número de precursores de neutrones atrasados, $n(t)$ es la densidad de neutrones, $\mathrm{C}_{\mathrm{i}}(\mathrm{t})$ son las concentraciones de precursores de neutrones atrasados, $q(t)$ es una fuente externa y $W_{1}(t), W_{2}(t), \ldots, W_{m+1}(t)$ son procesos de Wiener. Las matrices A y B están dadas de la siguiente forma:

$$
\mathrm{A}=\left[\begin{array}{ccccc}
\frac{\rho(\mathrm{t})-\boldsymbol{\beta}}{\Lambda} & \boldsymbol{\lambda}_{1} & \boldsymbol{\lambda}_{2} & \ldots & \boldsymbol{\lambda}_{\mathrm{m}} \\
\frac{\boldsymbol{\beta}_{1}}{\Lambda} & -\boldsymbol{\lambda}_{1} & \mathbf{0} & \ldots & \mathbf{0} \\
\frac{\boldsymbol{\beta}_{2}}{\Lambda} & \mathbf{0} & -\boldsymbol{\lambda}_{2} & \ldots & \mathbf{0} \\
\vdots & \vdots & \vdots & \ddots & \mathbf{0} \\
\frac{\boldsymbol{\beta}_{\mathrm{m}}}{\Lambda} & \mathbf{0} & \mathbf{0} & \ldots & -\boldsymbol{\lambda}_{\mathrm{m}}
\end{array}\right]
$$




$$
\widehat{\mathbf{B}}=\left[\begin{array}{ccccc}
\zeta & \mathbf{a}_{1} & \mathbf{a}_{2} & \ldots & \mathbf{a}_{\mathrm{m}} \\
\mathbf{a}_{1} & \mathbf{b}_{1,1} & \mathbf{b}_{1,2} & \ldots & \mathbf{b}_{1, \mathrm{~m}} \\
\mathbf{a}_{2} & \mathbf{b}_{2,1} & \mathbf{b}_{2,2} & \ldots & \mathbf{b}_{2, \mathrm{~m}} \\
\vdots & \vdots & \vdots & \ddots & \vdots \\
\mathbf{a}_{\mathrm{m}} & \mathbf{b}_{\mathrm{m}, 1} & \mathbf{b}_{\mathrm{m}, 2} & \cdots & \mathbf{b}_{\mathrm{m}, \mathrm{m}}
\end{array}\right]
$$

Donde

$$
\begin{aligned}
& \zeta=\left[\frac{\mathrm{n}(\mathrm{t})}{\mathrm{l}}\right]\left(-1-\rho(t)+2 \beta+v(1-\beta)^{2}\right)+\sum_{i=1}^{m} \lambda_{i} C_{i}(t)+\mathrm{q}(\mathrm{t}) \\
& a_{i}=\beta_{i}((1-\beta) \mathrm{v}-1)\left[\frac{\mathrm{n}(\mathrm{t})}{\mathrm{l}}\right]-\lambda_{i} C_{i}(t) \\
& b_{i, j}=\beta_{i} \beta_{j}\left[\frac{\mathrm{n}(\mathrm{t})}{\mathrm{l}}\right]+\delta_{i j} \lambda_{i} C_{i}(t) \\
& \delta_{i j}= \begin{cases}1 & \text { si } i=j \\
0 & \text { si } i \neq j\end{cases}
\end{aligned}
$$

Donde $v$ es el número promedio de neutrones generados por fisión, I es el tiempo de vida media del neutrón dentro del reactor, $\lambda_{i}$ es la constante de desintegración del i-ésimo grupo de precursores de neutrones atrasados, $\beta_{i}$ es la fracción efectiva de neutrones atrasados del i-ésimo grupo de precursores, $\beta$ es la fracción total de neutrones atrasados, $\rho$ es la reactividad y $\delta_{i j}$ es la delta de Kronecker. Es bueno observar que la ecuación (1) es la generalización de las ecuaciones de la cinética puntual, si $\hat{B}=0$ resulta el modelo determinista.

\section{MÉTODO PROPUESTO}

El método de Runge-Kutta implícito estocástico de orden 1.5 es un método numérico para solucionar sistemas de ecuaciones diferenciales estocásticas cuya solución también es un proceso estocástico, es un poco diferente a los métodos de Runge-Kutta determinísticos (Palma et al., 2010; Ahumada et al., 2017). En el caso multidimensional de un sistema de $\mathrm{m}+1$ ecuaciones, con $\mathrm{k}=1,2,3, \ldots, \mathrm{m}+1$, el método de Runge-Kutta implícito estocástico de orden 1.5 tiene la siguiente estructura (Kloeden et al., 2003):

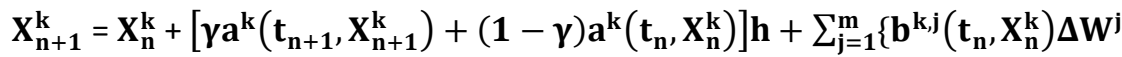

$$
\begin{aligned}
& \left.+\frac{1}{h}\left[b^{k, j}\left(t_{n+1}, X_{n}^{k}\right)-b^{k, j}\left(t_{n}, X_{n}^{k}\right)\right] I(0, j)+\frac{1}{2 \sqrt{h}}\left[a^{k}\left(t_{n}, \overline{\mathbf{Y}}_{+}^{\mathbf{j}}\right)-\mathbf{a}^{\mathbf{k}}\left(\mathbf{t}_{\mathbf{n}}, \overline{\mathbf{Y}}_{-}^{\mathbf{j}}\right)\right]\left[\mathbf{I}(\mathbf{j}, 0)-\frac{1}{2} \Delta \mathbf{W}^{\mathbf{j}} \mathbf{h}\right]\right\} \\
& +\frac{1}{2 \sqrt{\mathbf{h}}} \sum_{\mathbf{j}_{1}, j_{2}}^{\mathbf{m}}\left\{\mathbf{b}^{\mathbf{k}, \mathbf{j}_{2}}\left(\mathbf{t}_{\mathbf{n}+1}, \overline{\mathbf{Y}}_{+}^{\mathbf{j}}\right)-\mathbf{b}^{\mathbf{k}, \mathbf{j}_{2}}\left(\mathbf{t}_{\mathbf{n}}, \overline{\mathbf{Y}}_{-}^{\mathrm{j}}\right)\right\} \mathbf{I}\left(\mathbf{j}_{1}, \mathbf{j}_{2}\right) \\
& +\frac{1}{2 \mathbf{h}} \sum_{\mathbf{j}_{1}, \mathbf{j}_{2}}^{\mathrm{m}} \sum_{\mathbf{j}_{1}, j_{2}}^{\mathrm{m}}\left\{\mathbf{b}^{\mathbf{k}, \mathbf{j}_{2}}\left(\mathbf{t}_{\mathbf{n}+1}, \overline{\mathbf{Y}}_{+}^{\mathbf{j}_{1}}\right)-\mathbf{2} \mathbf{b}^{\mathbf{k}, \mathbf{j}_{2}}\left(\mathbf{t}_{\mathbf{n}}, \mathbf{X}_{\mathbf{n}}^{\mathbf{k}}\right)+\mathbf{b}^{\mathbf{k}, \mathbf{j}_{2}}\left(\mathbf{t}_{\mathbf{n}}, \overline{\mathbf{Y}}_{-}^{\mathbf{j}_{1}}\right)\right\} \mathbf{I}\left(\mathbf{0}, \mathbf{j}_{2}\right) \\
& +\frac{1}{2 h} \sum_{\mathbf{j}_{1}, j_{2}, j_{3}}^{\mathbf{m}}\left\{\mathbf{b}^{\mathbf{k}, j_{3}}\left(\mathbf{t}_{\mathbf{n}}, \overline{\boldsymbol{\phi}}_{+}^{\mathbf{j}_{1}, \mathbf{j}_{2}}\right)-\mathbf{b}^{\mathbf{k}, \mathbf{j}_{3}}\left(\mathbf{t}_{\mathbf{n}}, \overline{\boldsymbol{\phi}}_{-}^{\mathbf{j}_{1}, \mathbf{j}_{2}}\right)-\mathbf{b}^{\mathbf{k}, \mathbf{j}_{3}}\left(\mathbf{t}_{\mathbf{n}}, \overline{\mathbf{Y}}_{+}^{\mathbf{j}_{1}}\right)+\mathbf{b}^{\mathbf{k}, \mathbf{j}_{3}}\left(\mathbf{t}_{\mathbf{n}}, \overline{\mathbf{Y}}_{-}^{\mathbf{j}_{1}}\right)\right\} \mathbf{I}\left(\mathbf{j}_{1}, \mathbf{j}_{2}, \mathbf{j}_{3}\right)
\end{aligned}
$$

Donde

$$
\begin{aligned}
& \bar{Y}_{ \pm}^{j}=X_{n}^{j}+\frac{1}{m} a^{j}\left(t_{n}, X_{n}^{j}\right) h+\sum_{j=1}^{m} b^{j}\left(t_{n}, X_{n}^{j}\right) \sqrt{h} \\
& \bar{\phi}_{ \pm}^{j_{1}, j_{2}}=\bar{Y}_{+}^{j_{1}} \pm \sum_{j_{1}}^{m} b^{j_{1}}\left(t_{n}, \bar{Y}_{+}^{j_{2}}\right) \sqrt{h}
\end{aligned}
$$

Donde, $n=1,2,3, \ldots, N-1$. Siendo $\mathrm{N}$ el número de subdivisiones en el intervalo [to, T], el paso del tiempo para resolver las ecuaciones usando el método propuesto es dado por $h=t_{n+1}-t_{n}, X_{n}=X\left(t_{n}\right)$, el proceso de Wiener $\Delta \mathrm{W}^{\mathrm{i}}=\mathrm{W}_{\mathrm{n}+1}^{\mathrm{i}}-\mathrm{W}_{\mathrm{n}}^{\mathrm{i}}=\mathrm{h}^{1 / 2} \mathrm{n}\left(\mu, \sigma^{2}\right)$, siendo $\mathrm{n}\left(\mu, \sigma^{2}\right)$ una función de distribución normal con media $\mu=0$ y varianza $\sigma^{2}=1, \Delta Z^{j}=(1 / 2) h^{3 / 2}\left(U_{1}+(1 / 3)^{1 / 3} U_{2}\right)$ es normalmente distribuida que cumple $E\left(\Delta Z^{j}\right)=0$ y varianza $E\left(\left(\Delta Z^{j}\right)^{2}\right)=0$, esto se escribe como $\Delta Z^{\mathrm{j}} \sim \mathrm{n}\left(0,(1 / 3) \mathrm{h}^{3}\right)$ y tiene covarianza $E\left(\Delta \mathrm{W}^{\mathrm{j}} \Delta \mathrm{Z}^{\mathrm{j}}\right)=(1 / 2) \mathrm{h}^{2}$. Las integrales, I $(0, \mathrm{j})=\mathrm{h} \Delta \mathrm{W}^{\mathrm{j}}-\Delta \mathrm{Z}^{\mathrm{j}}$, $\mathrm{I}\left(\mathrm{j}_{1}, \mathrm{j}_{2}\right)=(1 / 2)\left(\Delta \mathrm{W}^{\mathrm{j}^{1}} \Delta \mathrm{W}^{\mathrm{j} 2}-\mathrm{h}\right), \mathrm{I}\left(\mathrm{j}_{1}, \mathrm{j}_{2}, \mathrm{j}_{3}\right)=(1 / 6) \Delta \mathrm{W}^{\mathrm{j}^{1}}\left(\Delta \mathrm{W}^{\mathrm{j}^{2}} \Delta \mathrm{W}^{\mathrm{j}}-3 \mathrm{~h}\right)$.

El método puede ser explicito, semi-implícito o totalmente implícito, esto dependiendo del valor que tome la constante $\gamma$, si $\gamma=0$ el método es explicito, si $0<\gamma<1$ el método es semi-implicito y si $\gamma=1$ el método es completamente implícito. A diferencia del método explicito cuya solución depende de valores del tiempo ya conocidos, el método implícito depende de un instante actual $t+h$. De esta manera para solucionar 
numéricamente la ecuación (1) de forma implícita utilizando el método propuesto en este trabajo se debe hacer $\mathrm{y}=1$ por lo tanto, resulta:

$$
\begin{aligned}
& \mathbf{X}_{\mathbf{n}+1}^{\mathrm{k}}=\mathbf{X}_{\mathbf{n}}^{\mathrm{k}}+\mathbf{a}^{\mathrm{k}}\left(\mathbf{t}_{\mathbf{n}+1}, \mathbf{X}_{\mathbf{n}+1}^{\mathrm{k}}\right) \mathbf{h}+\sum_{\mathbf{j}=1}^{\mathrm{m}}\left\{\mathbf{b}^{\mathrm{k}, \mathrm{j}}\left(\mathbf{t}_{\mathrm{n}}, \mathbf{X}_{\mathbf{n}}^{\mathrm{k}}\right) \Delta \mathbf{W}^{\mathbf{j}}\right. \\
& \left.+\frac{1}{h}\left[b^{k, j}\left(t_{n+1}, X_{n}^{k}\right)-\mathbf{b}^{k, j}\left(t_{n}, X_{n}^{k}\right)\right] \mathbf{I}(0, j)+\frac{1}{2 \sqrt{h}}\left[\mathbf{a}^{k}\left(t_{n}, \bar{Y}_{+}^{j}\right)-\mathbf{a}^{k}\left(t_{n}, \bar{Y}_{-}^{\mathbf{j}}\right)\right]\left[\mathbf{I}(\mathbf{j}, 0)-\frac{1}{2} \Delta \mathbf{W}^{\mathbf{j}} \mathbf{h}\right]\right\} \\
& +\frac{1}{2 \sqrt{\mathbf{h}}} \sum_{\mathbf{j}_{1}, \mathbf{j}_{2}}^{\mathbf{m}}\left\{\mathbf{b}^{\mathbf{k}, \mathbf{j}_{2}}\left(\mathbf{t}_{\mathbf{n}+1}, \overline{\mathbf{Y}}_{+}^{\mathbf{j}}\right)-\mathbf{b}^{\mathbf{k}, \mathbf{j}_{2}}\left(\mathbf{t}_{\mathbf{n}}, \overline{\mathbf{Y}}_{-}^{\mathbf{j}}\right)\right\} \mathbf{I}\left(\mathbf{j}_{1}, \mathbf{j}_{2}\right) \\
& +\frac{1}{2 h} \sum_{\mathbf{j}_{1}, j_{2}}^{\mathrm{m}_{2}} \sum_{\mathbf{j}_{1}, \mathbf{j}_{2}}^{\mathrm{m}}\left\{\mathbf{b}^{\mathbf{k}, \mathbf{j}_{2}}\left(\mathbf{t}_{\mathbf{n}+1}, \overline{\mathbf{Y}}_{+}^{\mathbf{j}_{1}}\right)-2 \mathbf{b}^{\mathbf{k}, \mathbf{j}_{2}}\left(\mathbf{t}_{\mathbf{n}}, \mathbf{X}_{\mathbf{n}}^{\mathrm{k}}\right)+\mathbf{b}^{\mathbf{k}, \mathbf{j}_{2}}\left(\mathbf{t}_{\mathbf{n}}, \overline{\mathbf{Y}}_{-}^{\mathrm{j}_{1}}\right)\right\} \mathbf{I}\left(\mathbf{0}, \mathbf{j}_{2}\right) \\
& +\frac{1}{2 h} \sum_{\mathbf{j}_{1}, j_{2}, j_{3}}^{\mathbf{m}}\left\{\mathbf{b}^{\mathbf{k}, \mathbf{j}_{3}}\left(\mathbf{t}_{\mathbf{n}}, \bar{\phi}_{+}^{\mathbf{j}_{1}, \mathbf{j}_{2}}\right)-\mathbf{b}^{\mathbf{k} \mathbf{j}_{3}}\left(\mathbf{t}_{\mathbf{n}}, \overline{\boldsymbol{\phi}}_{-}^{\mathbf{j}_{1}, \mathbf{j}_{2}}\right)-\mathbf{b}^{\mathbf{k}, \mathbf{j}_{3}}\left(\mathbf{t}_{\mathbf{n}}, \overline{\mathbf{Y}}_{+}^{\mathbf{j}_{1}}\right)+\mathbf{b}^{\mathbf{k}, \mathbf{j}_{3}}\left(\mathbf{t}_{\mathbf{n}}, \overline{\mathbf{Y}}_{-}^{\mathbf{j}_{1}}\right)\right\} \mathbf{I}\left(\mathbf{j}_{1}, \mathbf{j}_{2}, \mathbf{j}_{3}\right)
\end{aligned}
$$

Comparando la ecuación (12) con la ecuación (1) en forma discreta, se deduce:

$$
\begin{aligned}
& X_{n+1}^{k}=\hat{P}_{n+1} \\
& \mathrm{a}^{\mathrm{k}}\left(t_{n+1}, X_{n+1}^{k}\right)=A \hat{P}_{n+1}+\hat{Q}_{n+1} \\
& \mathrm{a}^{\mathrm{k}}\left(t_{n}, \bar{Y}_{+}^{j}\right)=A \bar{Y}_{+}^{j}+\hat{Q}_{n} \\
& \mathrm{a}^{\mathrm{k}}\left(t_{n}, \bar{Y}_{-}^{j}\right)=A \bar{Y}_{-}^{j}+\hat{Q}_{n} \\
& b^{k, j}\left(t_{n}, X_{n}^{k}\right)=\hat{B}^{1 / 2}\left(t_{n}, \hat{P}_{n}\right)
\end{aligned}
$$

Considerando las equivalencias de las ecuaciones (13-17) aplicadas al método de Runge-Kutta implícito de orden 1.5 dado por (12), el sistema a resolver es el siguiente:

$$
\begin{aligned}
& \widehat{\mathbf{P}}_{\mathbf{n}+1}=\mathbf{S}^{-1}\left(\widehat{\mathbf{P}}_{\mathbf{n}}+\sum_{\mathbf{j}=1}^{\mathrm{m}}\left\{\hat{\mathbf{B}} \widehat{\mathbf{B}}^{\frac{1}{2}}\left(\mathbf{t}_{\mathbf{n}}, \widehat{\mathbf{P}}_{\mathbf{n}}\right) \Delta \mathbf{W}^{\mathbf{j}}+\frac{1}{\mathbf{h}}\left[\widehat{\mathbf{B}}^{1 / 2}\left(\mathbf{t}_{\mathbf{n}+1}, \widehat{\mathbf{P}}_{\mathbf{n}}\right)-\widehat{\mathbf{B}}^{1 / 2}\left(\mathbf{t}_{\mathbf{n}}, \widehat{\mathbf{P}}_{\mathbf{n}}\right)\right] \mathbf{I}(\mathbf{0}, \mathbf{j})\right.\right. \\
& \left.+\frac{1}{2 \sqrt{\mathbf{h}}}\left[\mathbf{A} \overline{\mathbf{Y}}_{+}^{\mathbf{j}}-\mathbf{A} \overline{\mathbf{Y}}_{-}^{\mathbf{j}}\right]\left[\mathbf{I}(\mathbf{j}, \mathbf{0})-\frac{1}{2} \Delta \mathbf{W}^{\mathbf{j}} \mathbf{h}\right]\right\}+\frac{1}{2 \sqrt{\mathbf{h}}} \sum_{\mathbf{j}_{1}, \mathbf{j}_{2}}^{\mathbf{m}}\left\{\widehat{\mathbf{B}}^{1 / 2}\left(\mathbf{t}_{\mathbf{n}}, \overline{\mathbf{Y}}_{+}^{\mathbf{j}_{1}}\right)-\widehat{\mathbf{B}}^{1 / 2}\left(\mathbf{t}_{\mathbf{n}}, \overline{\mathbf{Y}}_{-}^{\mathbf{j}_{1}}\right)\right\} \mathbf{I}\left(\mathbf{j}_{1}, \mathbf{j}_{2}\right) \\
& +\frac{1}{2 \mathbf{h}} \sum_{\mathbf{j}_{1}, j_{2}}^{\mathrm{m}}\left\{\widehat{\mathbf{B}}^{1 / 2}\left(\mathbf{t}_{\mathbf{n}}, \overline{\mathbf{Y}}_{+}^{\mathbf{j}_{1}}\right)-2 \widehat{\mathbf{B}}^{1 / 2}\left(\mathbf{t}_{\mathbf{n}}, \widehat{\mathbf{P}}_{\mathbf{n}}\right)+\widehat{\mathbf{B}}^{1 / 2}\left(\mathbf{t}_{\mathbf{n}}, \overline{\mathbf{Y}}_{-}^{\mathbf{j}_{1}}\right)\right\} \mathbf{I}\left(\mathbf{0}, \mathbf{j}_{2}\right) \\
& \left.+\frac{1}{2 \mathbf{h}} \sum_{\mathbf{j}_{1}, \mathbf{j}_{2}, j_{3}}^{\mathbf{m}}\left\{\widehat{\mathbf{B}}^{1 / 2}\left(\mathbf{t}_{\mathbf{n}}, \overline{\boldsymbol{\phi}}_{+}^{\mathbf{j}_{1} \mathbf{j}_{2}}\right)-\widehat{\mathbf{B}}^{1 / 2}\left(\mathbf{t}_{\mathbf{n}}, \overline{\boldsymbol{\Phi}}_{-}^{\mathbf{j}_{1}, \mathbf{j}_{2}}\right)-\widehat{\mathbf{B}}^{1 / 2}\left(\mathbf{t}_{\mathbf{n}}, \overline{\mathbf{Y}}_{+}^{\mathbf{j}_{1}}\right)+\widehat{\mathbf{B}}^{\mathbf{1} / \mathbf{2}}\left(\mathbf{t}_{\mathbf{n}}, \overline{\mathbf{Y}}_{-}^{\mathbf{j}_{1}}\right)\right\} \mathbf{I}\left(\mathbf{j}_{1}, \mathbf{j}_{2}, \mathbf{j}_{3}\right)\right)
\end{aligned}
$$

La matriz $S$ y su matriz inversa $\mathrm{S}^{-1}$ vienen dadas por:

$$
\begin{gathered}
\mathrm{S}=\left(I-A_{n+1} h\right)=\left[\begin{array}{ccrcc}
1-\left(\rho\left(t_{n+1}\right)-\frac{\beta}{\Lambda}\right) h & -\lambda_{1} h & -\lambda_{2} h & \ldots & -\lambda_{m} h \\
-\left(\frac{\beta_{1}}{\Lambda}\right) h & 1+\lambda_{1} h & 0 & \ldots & 0 \\
-\left(\frac{\beta_{2}}{\Lambda}\right) h & 0 & 1+\lambda_{2} h & \ldots & 0 \\
\vdots & \vdots & \vdots & \ddots & \vdots \\
-\left(\frac{\beta_{m}}{\Lambda}\right) h & 0 & 0 & \ldots & 1+\lambda_{m} h
\end{array}\right] \\
S^{-1}=\left(I-A_{n+1} \mathrm{~h}\right)^{-1}=\left[\begin{array}{ccccc}
S_{1,1} & S_{1,2} & S_{1,3} & \ldots & S_{1, m} \\
S_{2,1} & S_{2,2} & S_{2,3} & \ldots & S_{2, m} \\
S_{3,1} & S_{3,2} & S_{3,3} & \ldots & S_{3, m} \\
\vdots & \vdots & \vdots & \ddots & \vdots \\
S_{m, 1} & S_{m, 2} & S_{m, 3} & \ldots & S_{m, m}
\end{array}\right]
\end{gathered}
$$

Los elementos matriciales de $S^{-1}$ están dados por:

$$
S_{1, j}=\frac{1}{\varepsilon_{n+1}}\left(1+\left(1-\delta_{1, j}\right)\left(\frac{\lambda_{j-1} h}{1+\lambda_{j-1} h}-1\right)\right), \quad \mathrm{j}=2,3,4, \ldots, \mathrm{m}+1
$$




$$
\begin{aligned}
& S_{i, j}=\frac{1}{1+\lambda_{j-1} h}\left(\delta_{i, j}+\frac{\beta_{j-1} h}{l} S_{1, j}\right), \quad \mathrm{i}=2,3,4, \ldots, \mathrm{m}+1 ; \mathrm{j}=1,2,3, \ldots, \mathrm{m}+1 \\
& \varepsilon_{n+1}=1-\left[\frac{\rho\left(t_{n+1}-\beta\right)}{l}\right] h-\frac{h^{2}}{l}\left[\sum_{i=1}^{m} \frac{\lambda_{i} \beta_{i}}{1+\lambda_{j-1} h}\right]
\end{aligned}
$$

En este trabajo se utiliza la matriz de covarianza (Suescún-Díaz et al., 2018), la cual es obtenida partiendo de la teoría de difusión de una velocidad sujeta a la condición inicial aplicando el método de neutrones pulsados el cual se explica, que para un pulso rápido la evolución temporal de la densidad de neutrones $n(t)$ viene dada por la cinética de neutrones instantáneos de la forma:

$$
\frac{d n(t)}{d t}=\left(\frac{\rho-\beta}{\Lambda}\right) n(t)
$$

Donde se satisface la relación

$$
\frac{1}{l}=\left(\frac{\rho-\beta}{\Lambda}\right)
$$

Donde $\wedge$ el tiempo de generación de neutrones y es definido por:

$$
\Lambda=\frac{l}{k}
$$

Siendo k el factor de multiplicación, reemplazando la ecuación (25) en las ecuaciones (5-7) se obtiene la modificación a la matriz de covarianza dada por la ecuación (4), mediante el método de neutrones pulsados, la matriz de covarianza queda de la siguiente manera :

$$
\widehat{B}^{*}=\left[\begin{array}{ccccc}
\zeta^{*} & \boldsymbol{a}_{1}^{*} & \boldsymbol{a}_{2}^{*} & \ldots & \boldsymbol{a}_{m}^{*} \\
\boldsymbol{a}_{1}^{*} & \boldsymbol{b}_{1,1}^{*} & \boldsymbol{b}_{1,2}^{*} & \ldots & \boldsymbol{b}_{1, m}^{*} \\
\boldsymbol{a}_{2}^{*} & \boldsymbol{b}_{2,1}^{*} & \boldsymbol{b}_{2,2}^{*} & \ldots & \boldsymbol{b}_{2, m}^{*} \\
\vdots & \vdots & \vdots & \ddots & \vdots \\
\boldsymbol{a}_{m}^{*} & \boldsymbol{b}_{m, \mathbf{1}}^{*} & \boldsymbol{b}_{m, 2}^{*} & \cdots & \boldsymbol{b}_{m, m}^{*}
\end{array}\right]
$$

Donde

$$
\begin{aligned}
& \zeta^{*}=\left[\frac{\rho-\beta}{\Lambda}\right]\left(-1-\rho(t)+2 \beta+v(1-\beta)^{2}\right) n(t)+\sum_{i=1}^{m} \lambda_{i} C_{i}(t)+\mathrm{q}(\mathrm{t}) \\
& a_{i}^{*}=\beta_{i}((1-\beta) \mathrm{v}-1)\left[\frac{\rho-\beta}{\Lambda}\right] n(t)-\lambda_{i} C_{i}(t) \\
& b_{i, j}^{*}=\beta_{i} \beta_{j}\left[\frac{\rho-\beta}{\Lambda}\right] n(t)+\delta_{i j} \lambda_{i} C_{i}(t)
\end{aligned}
$$

\section{RESULTADOS}

En esta sección se presentan algunos de los resultados de los diferentes experimentos numéricos realizados utilizando el método de Runge-Kutta implícito de orden 1.5 dado por la ecuación (12), esto se hizo para el cálculo de las ecuaciones estocásticas de la cinética puntual utilizando la matriz de covarianza representada en la ecuación (4) y de igual manera con la representada por la ecuación (27), estos resultados se comparan con otros métodos reportados en la literatura como PCA y Monte Carlo (Hayes y Allen, 2005), Euler-Maruyama explícito y Taylor 1.5 (Ray, 2012), AEM (Nahla y Edress, 2016a), ESM (Nahla y Edress, 2016b), Doble DDM (Da silva et al., 2016), Euler-Maruyama implícito (Suescún-Díaz et al., 2018) y el método determinista de Runge-Kutta. Se realizaron varios experimentos utilizando diferentes valores de reactividad constante, considerando 5000 trayectorias Brownianas para cada experimento excepto para el último experimento.

El primer experimento numérico considera el caso de un precursor y una inserción de reactividad con un valor de $\rho=-1 / 3$, con un tiempo de generación $\Lambda=(2 / 3) \mathrm{s}$, la constante de decaimiento $\lambda_{1}=0.1 \mathrm{~s}^{-1}$, la fracción de neutrones atrasados $\beta=\beta_{1}=0.05$, el promedio de neutrones generados por fisión $v=2.5$, se considera una fuente externa de neutrones con un valor $q=200 \mathrm{~s}^{-1}$, con la condición inicial $\hat{P}_{0}=[400,300]^{\top}$, con un número total de $\mathrm{N}=40$ subdivisiones en el tiempo en el intervalo [0,2]s. La Tabla 1 muestra los resultados para el primer experimento utilizando la matriz de covarianza dada en la ecuación (4) y con la propuesta dada por la ecuación (27), de igual forma se compara con los resultados de los otros métodos reportados en la literatura. En la Figura 1 se muestra la evolución temporal de la densidad de la población de neutrones para el primer experimento. La solución determinística es una constate, las soluciones estocásticas son claramente diferenciadas en su comportamiento siguiendo las dos rutas un movimiento Browniano. 
Tabla 1: Comparación de los métodos para el caso de un precursor y reactividad $\rho=-1 / 3$

\begin{tabular}{|c|c|c|c|c|}
\hline Método & $E(n(2))$ & $\sigma(n(2))$ & $E\left(\Sigma C_{i}(2)\right)$ & $\sigma\left(\Sigma C_{i}(2)\right)$ \\
\hline Monte Carlo & 400.03 & 27.31 & 300.00 & 7.81 \\
\hline $\mathrm{PCA}$ & 395.32 & 27.41 & 300.67 & 8.36 \\
\hline Euler-Maruyama & 412.23 & 34.39 & 315.96 & 8.27 \\
\hline Taylor 1.5 & 412.10 & 34.52 & 315.93 & 8.32 \\
\hline AEM & 396.28 & 31.21 & 300.42 & 7.96 \\
\hline ESM & 396.62 & 0.92 & 300.39 & 0.00 \\
\hline DDDM & 402.35 & 28.61 & 305.84 & 7.92 \\
\hline EM implícito $(\hat{B})$ & 399.71 & 31.43 & 299.77 & 7.94 \\
\hline EM implícito $\left(\hat{\mathrm{B}}^{*}\right)$ & 399.99 & 0.54 & 299.87 & 6.84 \\
\hline RK implícito $1.5(\hat{B})$ & 399.45 & 30.60 & 299.87 & 7.99 \\
\hline RK implícito $1.5\left(\hat{B}^{*}\right)$ & 400.01 & 0.84 & 299.92 & 7.69 \\
\hline $\mathrm{RKO} O\left(h^{4}\right)$ & 400.00 & - & 300.00 & - \\
\hline
\end{tabular}

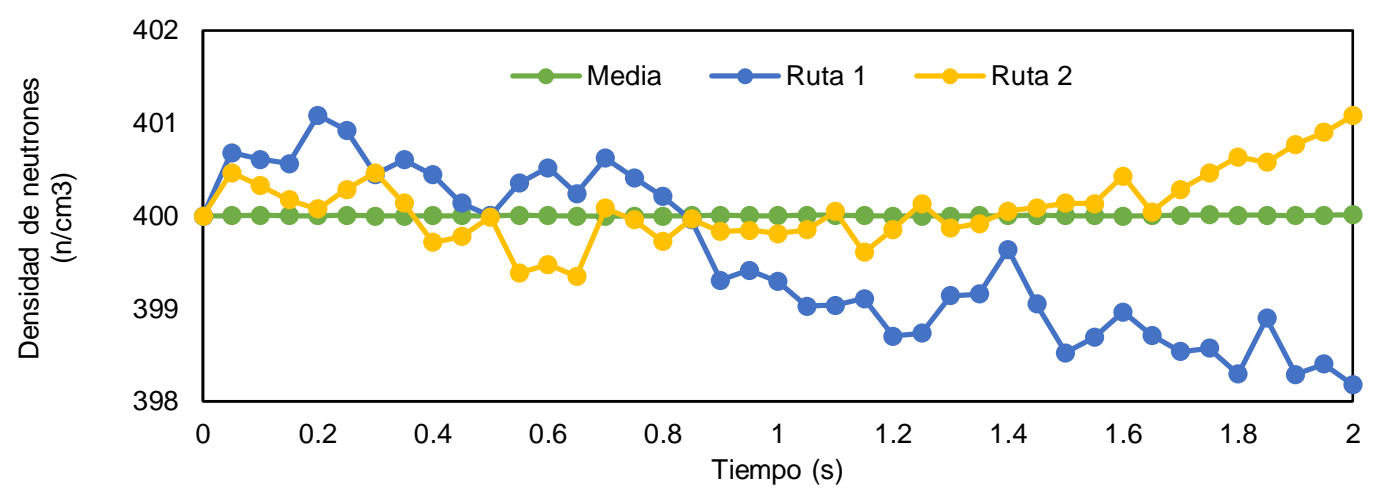

Fig.1: Densidad de población de neutrones en función del tiempo con reactividad $\rho=-1 / 3$

En el segundo y tercer experimento numérico se consideran seis grupos de precursores de neutrones atrasados con valores de reactividad de $\rho=0.003$ y $\rho=0.007$ respectivamente, con los parámetros físicos siguientes: el tiempo de generación de neutrones instantáneos $\Lambda=2^{*} 10^{-5} \mathrm{~s}$, el número promedio de neutrones instantáneos debido a la fisión $\mathrm{v}=2.5$, las constantes de decaimiento para cada grupo de precursores son $\lambda_{i}=[0.0127,0.0317,0.115,0.311,1.4,3.87] \mathrm{s}^{-1}$ la fracción de neutrones atrasados $\beta_{i}=[0.000266,0.001491$, $0.001316,0.002849,0.000896,0.000182]$, la fracción total de neutrones atrasados $\beta=0.007$, la fuente de neutrones $\mathrm{q}=0 \mathrm{~s}^{-1}, \mathrm{~N}=40$ subdivisiones en el tiempo. Para el segundo y tercer experimento numérico se realizan para un intervalo de tiempo de $[0,0.1]$ s y $[0,0.001]$ s respectivamente, con condición inicial $\hat{P}_{0}=100[1$, $\left.\beta_{1} /\left(\Lambda \lambda_{1}\right), \beta_{2} /\left(\Lambda \lambda_{2}\right), \beta_{3} /\left(\Lambda \lambda_{3}\right), \beta_{4} /\left(\Lambda \lambda_{4}\right), \beta_{5} /\left(\Lambda \lambda_{5}\right), \beta_{6} /\left(\Lambda \lambda_{6}\right)\right]$. Las Tablas 2-3 muestran una comparación con los métodos reportados en la literatura para valores de reactividad $\rho=0.003$ y $\rho=0.007$ respectivamente, se muestra el valor esperado y la desviación estándar de la densidad de neutrones y la concentración de precursores de neutrones atrasados para $t=0.1 \mathrm{~s} \mathrm{y} t=0.001 \mathrm{~s}$ respectivamente, se realizaron 5000 trayectorias Brownianas para cada experimento. Los resultados indican que el método propuesto tiene valores medios muy cercanos a los valores determinísticos dados por el método $\mathrm{RK} O\left(\mathrm{~h}^{4}\right)$, el valor en la desviación estándar es inferior cuando se consideran las dos clases de matrices de covarianzas indicadas por $\hat{B}$ y $\hat{B}^{*}$. En las Figuras 2-3 se muestran la evolución temporal para la población de neutrones para $\rho=0.003$ y $\rho=0.007$ respectivamente, considerando dos muestras cuyos valores oscilan alrededor de la curva media dada por las 5000 trayectorias Brownianas. Los resultados indican que el método propuesto es bastante preciso.

Tabla 2: Comparación de los métodos con seis precursores y reactividad $\rho=0.003$

\begin{tabular}{|c|c|c|c|c|}
\hline Método & $E(n(0.1))$ & $\sigma(n(0.1))$ & $E\left(\sum C_{i}(0.1)\right)$ & $\sigma\left(\sum C_{i}(0.1)\right)$ \\
\hline Monte Carlo & 183.04 & 168.79 & 447800 & 1495.70 \\
\hline$\overline{P C A}$ & 186.31 & 164.16 & 449100 & 1917.20 \\
\hline Euler-Maruyama & 208.60 & 255.95 & 449800 & 1233.38 \\
\hline Taylor 1.5 & 199.41 & 168.55 & 449700 & 1218.82 \\
\hline AEM & 186.30 & 164.14 & 449000 & 1911.91 \\
\hline ESM & 179.93 & 10.56 & 448900 & 94.75 \\
\hline DDDM & 187.05 & 167.83 & 448800 & 1475.60 \\
\hline EM implícito ( $\hat{B})$ & 178.27 & 165.11 & 448860 & 1253.60 \\
\hline EM implícito $\left(\hat{\mathrm{B}}^{*}\right)$ & 179.94 & 0.22 & 448880 & 60.43 \\
\hline RK implícito 1.5 (B) & 181.66 & 139.02 & 448878 & 1623.20 \\
\hline RK implícito $1.5\left(\hat{B}^{*}\right)$ & 180.00 & 1.98 & 448880 & 55.41 \\
\hline $\mathrm{RK} \mathrm{O}\left(h^{4}\right)$ & 179.95 & - & 448878 & - \\
\hline
\end{tabular}


Tabla 3: Comparación de los métodos con seis precursores y reactividad $\rho=0.007$

\begin{tabular}{|l|c|c|c|c|}
\hline Método & $E(n(0.001))$ & $\sigma(n(0.001))$ & $E\left(\sum C_{i}(0.001)\right)$ & $\sigma\left(\sum C_{i}(0.001)\right)$ \\
\hline Monte Carlo & 135.67 & 93.38 & 446400 & 16.23 \\
\hline PCA & 134.55 & 91.24 & 446400 & 19.44 \\
\hline Euler-Maruyama & 139.57 & 92.04 & 446300 & 6.07 \\
\hline Taylor 1.5 & 139.57 & 92.05 & 446300 & 18.34 \\
\hline AEM & 134.54 & 91.23 & 446400 & 19.24 \\
\hline ESM & 134.96 & 6.85 & 446400 & 2.53 \\
\hline DDDM & 135.86 & 93.21 & 446300 & 17.85 \\
\hline EM implícito $(\hat{\mathrm{B}})$ & 134.02 & 93.27 & 446360 & 18.78 \\
\hline EM implícito $\left(\hat{\mathrm{B}}^{*}\right)$ & 134.92 & 5.97 & 446360 & 6.07 \\
\hline RK implícito 1.5 $(\hat{\mathrm{B}})$ & 134.35 & 71.49 & 446360 & 16.19 \\
\hline RK implícito 1.5 $\left(\hat{\mathrm{B}}^{*}\right)$ & 134.99 & 2.92 & 446360 & 6.93 \\
\hline RK O $\left(h^{4}\right)$ & 135.00 & - & 446360 & - \\
\hline
\end{tabular}

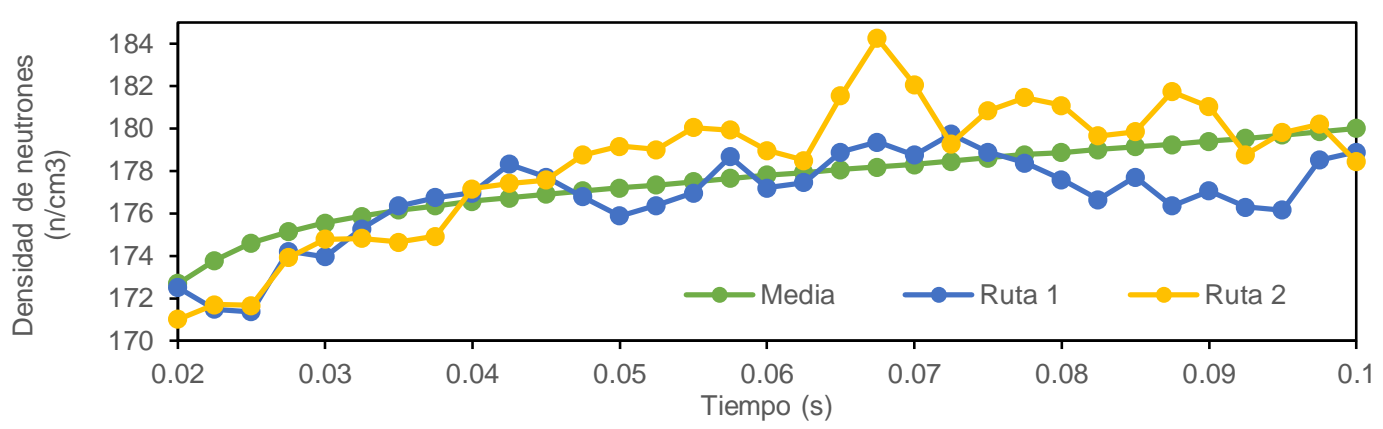

Fig.2: Densidad de población de neutrones para seis grupos de precursores con reactividad $\rho=0.003$

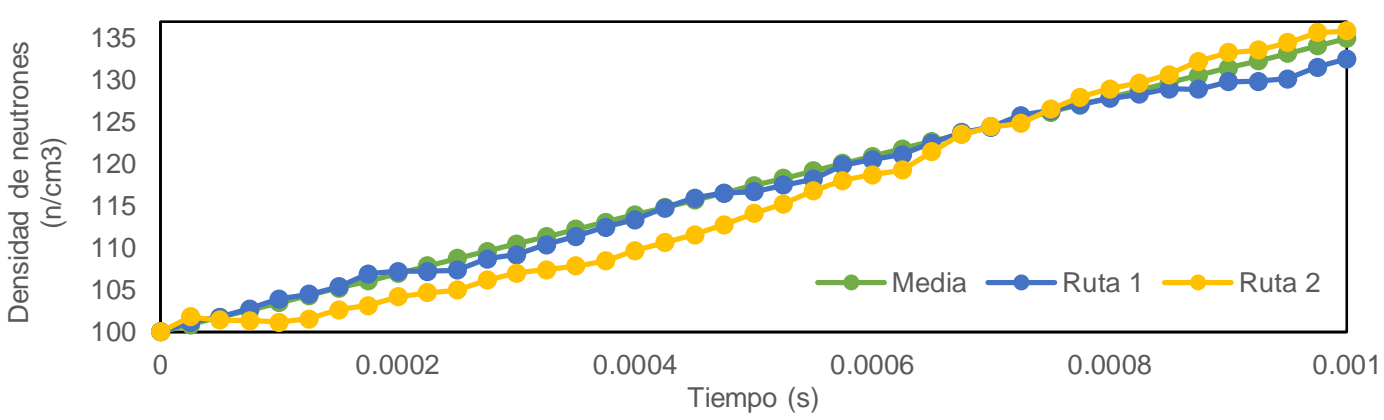

Fig.3: Densidad de población de neutrones para seis grupos de precursores con reactividad $\rho=0.007$

El último experimento numérico se comparan los resultados para un reactor real TRIGA (Ayyoubzadeh y Vosoughi, 2014 ; El-Beltagy y Noor, 2019). Es un reactor térmico alimentado con uranio-235 con los siguientes parámetros: tiempo de generación de neutrones $\Lambda=0.0001(s)$, constante de decaimiento $\lambda_{1}=0.077\left(s^{-1}\right)$, fracción de precursores de neutrones retardados $\beta=0.0079$, promedio de neutrones generados por evento de fisión $v=2.432$, fuente externa de neutrones $q(t)=10000$, para un valor de reactividad $\rho=0.003$, condición inicial $n(0)=0, C(0)=0$. Esta simulación se realiza con 100 iteraciones en un intervalo de tiempo de $[0,0.1]$ s, empleando 100, 1000 y 10000 movimientos Brownianos (MB) con el método propuesto RungeKutta implícito de orden 1.5 con matrices de covarianzas $\hat{B}$ y $\hat{B}^{*}$. Se presentan en la Tabla 4 y evidencian como el esquema de Runge-Kutta implícito 1.5 provee buenas aproximaciones con la matriz de covarianza $\hat{\mathrm{B}}^{*}$, tanto en valores medios como en baja desviación estándar con solo $100 \mathrm{MB}$, respecto a los valores reportados usando los métodos Monte Carlo, SPCA y SSPK con 10000 procesos de Wiener. Las pequeñas fluctuaciones hacen que el modelo estocástico de la cinética puntual describa de forma más real el comportamiento de la población de neutrones en un reactor nuclear.

Tabla 4: Comparación de resultados para un reactor TRIGA

\begin{tabular}{|c|c|c|c|c|}
\hline Método & $E(n(0.1))$ & $\sigma(n(0.1))$ & $E\left(\sum C_{i}(0.1)\right)$ & $\sigma\left(\sum C_{i}(0.1)\right)$ \\
\hline Monte Carlo & 199.15 & 152.63 & 1254.50 & 613.94 \\
\hline SPCA & 204.52 & 174.03 & 1294.00 & 620.68 \\
\hline SSPK & 208.14 & 174.30 & 1293.20 & 622.12 \\
\hline EWH & 204.11 & 171.14 & 1285.74 & 623.28 \\
\hline RK implícito $1.5(\hat{B}) 1000 \mathrm{MB}$ & 195.65 & 158.66 & 1310.90 & 628.07 \\
\hline RK implícito 1.5 (B) $5000 \mathrm{MB}$ & 201.92 & 169.03 & 1291.00 & 623.32 \\
\hline RK implícito $1.5\left(\hat{\mathrm{B}}^{\star}\right) 100 \mathrm{MB}$ & 204.04 & 0.14 & 1281.60 & 16.36 \\
\hline
\end{tabular}




\section{CONCLUSIONES}

El método de Runge-Kutta implícito de orden 1.5 es utilizado para solucionar numéricamente las ecuaciones de la cinética puntual estocástica, las cuales modelan el comportamiento de la densidad de neutrones y la concentración de precursores. Una ventaja de este método de alta precisión es que no utiliza derivadas como las presentadas en el método de Taylor estocástico 1.5, permitiendo su implementación en una forma más sencilla. Otra ventaja es que no necesita invertir alguna matriz, aunque el método sea implícito, ya que la inversión se realiza analíticamente disminuyendo el costo computacional. Sin embargo, una desventaja es el alto número de evaluaciones necesarias para conseguir esa alta precisión, aunque este esfuerzo computacional se vea disminuido por considerar un menor número de trayectorias Brownianas. El aporte en este trabajo es la implementación del método en el campo de la física de reactores nucleares, utilizando la matriz de covarianza tradicional y la modificada, esta última, la cual incorpora la teoría de difusión de neutrones de una velocidad considerando el método de neutrones pulsados. Estas consideraciones permiten obtener una buena aproximación en los valores medios y una disminución en la desviación estándar en la población de neutrones y en la concentración de precursores como mostrado en los diferentes experimentos numéricos, presentados en este trabajo que están muy acorde con los resultados encontrados en la literatura, permitiendo que el método propuesto sea un método alternativo para resolver numéricamente las ecuaciones de la cinética puntual estocástica.

\section{REFERENCIAS}

Ahumada, L.M., Audivet, C.P., Bula, A.J., y Sanjuan, M.E., Comparación entre Soluciones Numéricas de un Modelo Matemático de Combustión Teórica y Variación de la Conductividad del Cuesco de Palma en un Reactor de Lecho Fijo, https://doi.org/10.4067/S0718-07642017000300018, Inf. Tecnol., 28(3), 171-184 (2017)

Ayyoubzadeh, S.M., y Vosoughi, N., An Alternative Stochastic Formulation for the Point Reactor, https://doi.org/10.1016/j.anucene.2013.09.013, Ann. Nucl. Energy., 63, 691-695 (2014)

El-Beltagy, M., y Noor, A., Analysis of the Stochastic Point Reactor Using Wiener-Hermite Expansion, https://doi.org/10.1016/j.anucene.2019.06.022, Ann. Nucl. Energy., 134, 250-257 (2019)

Gordon, T.L., Cooling, C.M., Williams, M.M.R., y Eaton, M.D., Numerical Comparison of Mathematical and Computational Models for the Simulation of Stochastic Neutron Kinetics Problems,

https://doi.org/10.1016/j.anucene.2021.108161, Ann. Nucl. Energy., 157, 108161 (2021)

Hayes, J.G., y Allen, E.J., Stochastic Point Kinetic Equations in Nuclear Reactor Dynamics, https://doi.org/10.1016/..anucene.2004.11.009, Ann. Nucl. Energy., 32, 572-587 (2005)

Kloeden, P.E., Platen, E., y Schurz, H., Numerical Solution of SDE Through Computer Experiments, Springer-Verlag, Heidelberg, Alemania (2003)

Nahla, A.A., y Edress, A.M., Analytical Exponential Model for Stochastic Point Kinetic Equations via Eigenvalues and Eigenvectors, https://doi.org/10.1007/s41365-016-0025-6, Nucl. Sci.Technol., 27, 19-27 (2016a)

Nahla, A.A., y Edress, A.M., Efficient Stochastic Model for the Point Kinetics Equations, https://doi.org/10.1080/07362994.2016.1159519, Stochast. Analy. Appl., 34, 598-609 (2016b)

Nayak, S., y Chakraverty, S., Numerical Solution of Stochastic Point-Kinetics Neutron Equation with Fuzzy Parameters, https://doi.org/10.13182/NT15-63, Nucl. Technol., 193, 444-456 (2016)

Palma, J.A., Barbosa, J.M.A., y Silva, A.A., Simulación Numérica de la Propagación de una Fisura en un Material Degradado por efecto de la Fragilización por Hidrógeno, https://doi.org/10.4067/S0718-07642010000200009, Inf. Tecnol., 21(2), 65-76 (2010)

Ray, S.S., Numerical Simulation of Stochastic Point Kinetic Equations in the Dynamical System of Nuclear Reactor, https://doi.org/10.1016/j.anucene.2012.05.022, Ann. Nucl. Energy., 49, 154-159 (2012)

Ray, S.S., y Patra, A., Numerical Simulation for Stochastic Point Kinetic Equations with Sinusoidal Reactivity in Dynamical System of Nuclear Reactor, https://doi.org/10.1504/IJNEST.2013.052165, Int. J. Nucl. Sci. Technol., 7, 231$242(2013)$

Singh, S.S., y Ray, S., On the Comparison of two Split-Step Methods for the Numerical Simulation of Stochastic Point Kinetics Equations in Presence of Newtonian Temperature Feedback Effects, https://doi.org/10.1016/j.anucene.2017.08.001, Ann. Nucl. Energy., 110, 865-873 (2017)

Stacey, W.M., Nuclear Reactor Physics, 3a edición, Wiley-VCH, Verlag, Weinheim, Alemania (2018)

Suescún-Díaz, D., Oviedo-Torres, Y.M., y Giron-Cruz, L.E., Solution of the Stochastic Point Kinetics Equations Using the Implicit Euler-Maruyama Method, https://doi.org/10.1016/j.anucene.2018.03.013, Ann. Nucl. Energy., 117, 45-52 (2018)

Wollmann da Silva, M., Vasques, R., Bodmann, B.E.J., y Vilhena, M.T., A Nonstiff Solution for the Stochastic Neutron Point Kinetics Equations, https://doi.org/10.1016/j.anucene.2016.06.026, Ann. Nucl. Energy., 97, 47-52 (2016) 